\title{
Dynamic and reversible DNA methylation changes induced by genome separation and merger of polyploid wheat
}

\author{
Jingya Yuan ${ }^{1}$, Wu Jiao ${ }^{1}$, Yanfeng Liu', Wenxue Ye ${ }^{1}$, Xiue Wang ${ }^{1}$, Bao Liü, Qingxin Song ${ }^{1 *}$ and Z. Jeffrey Chen ${ }^{1,3^{*}}$ (D)
}

\begin{abstract}
Background: Wheat is a powerful genetic model for studying polyploid evolution and crop domestication. Hexaploid bread wheat was formed by two rounds of interspecific hybridization and polyploidization, processes which are often accompanied by genetic and epigenetic changes, including DNA methylation. However, the extent and effect of such changes during wheat evolution, particularly from tetraploid-to-hexaploid wheat, are currently elusive.

Results: Here we report genome-wide DNA methylation landscapes in extracted tetraploid wheat (ETW, AABB), natural hexaploid wheat (NHW, AABBDD), resynthesized hexaploid wheat (RHW, AABBDD), natural tetraploid wheat (NTW, $A A B B)$, and diploid (DD). In the endosperm, levels of DNA methylation, especially in $\mathrm{CHG}(H=\mathrm{A}, \mathrm{T}$, or $\mathrm{C})$ context, were dramatically decreased in the ETW relative to natural hexaploid wheat; hypo-differentially methylated regions (DMRs) $(850,832)$ were 24 -fold more than hyper-DMRs $(35,111)$. Interestingly, those demethylated regions in ETW were remethylated in the resynthesized hexaploid wheat after the addition of the D genome. In ETW, hypo-DMRs correlated with gene expression, and TEs were demethylated and activated, which could be silenced in the hexaploid wheat. In NHW, groups of TEs were dispersed in genic regions of three subgenomes, which may regulate the expression of TEassociated genes. Further, hypo-DMRs in ETW were associated with reduced H3K9me2 levels and increased expression of histone variant genes, suggesting concerted epigenetic changes after separation from the hexaploid.
\end{abstract}

Conclusion: Genome merger and separation provoke dynamic and reversible changes in chromatin and DNA methylation. These changes correlate with altered gene expression and TE activity, which may provide insights into polyploid genome and wheat evolution.

Keywords: Extracted tetraploid wheat, DNA methylation, Transposon, Genomics, Polyploidy, Wheat evolution

\section{Background}

Polyploidy is a prominent feature for the evolution of some animals and all flowering plants, including most important crops such as wheat, cotton, and canola [1-3]. The common occurrence of polyploidy suggests an advantage for having additional genetic materials for diversification of polyploid plants and domestication of polyploid crops.

* Correspondence: qxsong@njau.edu.cn; zjchen@austin.utexas.edu ${ }^{1}$ State Key Laboratory of Crop Genetics and Germplasm Enhancement, Nanjing Agricultural University, 1 Weigang Road, Nanjing 210095, China Full list of author information is available at the end of the article
Interspecific hybridization in cotton is accompanied with genetic and epigenetic changes including DNA methylation, many of which are maintained as stable epialleles among five allotetraploid cotton species during evolution and two cultivated cottons during domestication [4]. In addition to DNA methylation, genome-wide histone modifications determine expression bias of homeologous genes in alloptetraploid cotton [5], and long-noncoding RNAs (lncRNAs) are predominantly transcribed from transposable element

(c) The Author(s). 2020 Open Access This article is licensed under a Creative Commons Attribution 4.0 International License, which permits use, sharing, adaptation, distribution and reproduction in any medium or format, as long as you give appropriate credit to the original author(s) and the source, provide a link to the Creative Commons licence, and indicate if changes were made. The images or other third party material in this article are included in the article's Creative Commons licence, unless indicated otherwise in a credit line to the material. If material is not included in the article's Creative Commons licence and your intended use is not permitted by statutory regulation or exceeds the permitted use, you will need to obtain permission directly from the copyright holder. To view a copy of this licence, visit http://creativecommons.org/licenses/by/4.0/. The Creative Commons Public Domain Dedication waiver (http://creativecommons.org/publicdomain/zero/1.0/) applies to the data made available in this article, unless otherwise stated in a credit line to the data. 
(TE) regions that are demethylated in a cotton interspecific hybrid [6]. This is consistent with the nonadditive expression of microRNAs (miRNAs) and small interfering RNAs (siRNAs) in Arabidopsis allotetraploids as a response to "genome shock" during interspecific hybridization [7].

The evolution of hexaploid bread wheat (Triticum aestivum L.) coincided with human civilization through two rounds of interspecific hybridization and whole-genome duplication (WGD) [8, 9], involving mechanisms of correct pairing between homoeologous chromosomes [10]. The common wheat $(2 n=6 x=42$, AABBDD) was formed $\sim 8000$ 10,000 years ago by crossing between tetraploid wheat (Triticum turgidum L., AABB) and a goatgrass species (Aegilops tauschii, DD), coupled with genome doubling [8]. The A, B, and $\mathrm{D}$ subgenomes remain largely intact, with only a few homoeologous chromosome translocations [11, 12]. The tetraploid genomic (AABB) component could be extracted by hybridization between a natural hexaploid wheat (NHW, $\mathrm{AABBDD}$ ) and a natural tetraploid wheat (NTW, AABB) with nine generations of backcrossing using NHW as the recurrent parent, followed by three generations of selfpollination to exclude $\mathrm{D}$ chromosome and stabilize the genotype [13] (Fig. 1a). The extracted tetraploid wheat (ETW, $\mathrm{AABB}$ ) is dwarfed and displays severe developmental abnormalities compared to natural tetraploid and hexaploid wheat, but the chromosomes pair and segregate normally $[13,14]$. The abnormal phenotypes are recovered in the resynthesized allohexaploid wheat (RHW) formed by hybridization between ETW and a D-genome diploid (Ae. tauschii) $[13,14]$. Previous studies show that ETW has lower levels of DNA methylation using 5-methylC antibodies [15] and more differentially expressed genes [14], compared to the natural tetraploid wheat (NTW). However, it is unknown how DNA methylation changes from the separation of the ETW (AABB component) in the natural hexaploid wheat to the formation of resynthesized hexaploid wheat.

In this study, we performed RNA sequencing (RNAseq) and whole-genome bisulfite sequencing (BS-seq) in the endosperm of ETW (AABB), the natural hexaploid wheat ( $T$. aestivum $L$. cv. Canthach), natural tetraploid wheat (T. turgidum L. subsp. durum), Ae. tauschii subsp. strangulata (line RL5288, DD), and the resynthesized hexaploid wheat. We found genome-wide reduction of DNA methylation in the ETW and remethylation of these demethylated regions in the resynthesized hexaploid wheat after addition of the D subgenome, which are accompanied by changes in gene and TE expression. DNA demethylation in ETW coincides with reduced H3K9me2 levels and increased expression of histone genes. These results suggest that genome separation and merger promote dynamic and reversable epigenetic and gene expression changes in the evolution of polyploid wheat.

\section{Results}

\section{Phenotypic, karyotypic, and gene expression changes in} ETW

ETW was produced by hybridization between a natural hexaploid wheat (NHW, AABBDD) and a natural tetraploid wheat (NTW, AABB). The resulting pentaploid (AABBD) was backcrossed to NHW for nine generations, followed by three generations of self-pollination to stabilize the AABB karyotype and exclude D chromosomes (Fig. 1a) [13]. After separation of ETW (AABB) from the DD in NHW (AABBDD), ETW displays severe phenotypic defects, including dwarfed plants, compacted spikes, shriveled seeds, decreased starch content, smaller starch granule, and reduced fertility [14] (Fig. 1a and Additional file 1: Figure S1). Interestingly, the seed size and other abnormal phenotypes were recovered in the resynthesized allohexaploid wheat (RHW) by addition of the D subgenome (Ae. tauschii) to ETW (Fig. 1b). Fluorescent in situ hybridization (FISH) analysis showed stable karyotypes of homoeologous chromosomes in ETW relative to NHW and NTW (Additional file 1: Figure S2), which is consistent with the notion that ETW is highly (>99.8\%) identical to the AB subgenomes of its NHW donor after the seventh [13] or ninth backcross [14] (Fig. 1a).

The phenotypic variation such as seed size in ETW could be related to gene expression changes in developing seeds. In wheat, the development of starchy endosperm and aleurone layer begins from $\sim 6$ days after pollination (DAP) and is critical to seed development [16]. At this stage, seed size and shape were noticeably smaller in ETW than in NHW and RHW (Additional file 1: Figure S1b). To determine gene expression changes during seed development, we performed RNAseq analysis in the endosperm (6 DAP) of ETW, NHW, RHW, NTW (T. durum), and DD diploid (Ae. tauschii) (Additional file 2: Table S1). Between ETW and NHW, 4947 and 4537 genes were up- and downregulated, respectively (Additional file 2: Table S2). Of these differentially expressed genes (DEGs), the majority (65 70\%) were recovered in the RHW after the addition of the D genome to ETW (Fig. 1c). Gene ontology (GO) analysis showed overrepresentation of the DEGs in metabolism and development-related processes, including protein metabolism and embryo development (Fig. 1d), suggesting a potential role of these pathway genes in seed phenotypic changes in ETW after separation from NHW. For example, in Arabidopsis and rice, MADS-box genes are expressed in developing seeds and play important roles in reproductive development $[17,18]$. Knockdown of OsMADS7 and OsMADS8 could induce variable floral organs in rice [19]. These MADS-box genes such as TaMADS8 and TaMADS7 were repressed in ETW and derepressed in RHW after adding back the D subgenome (Fig. 1e), indicating a possible role of MADS-box genes in seed size reduction in ETW. Consistent with low starch 


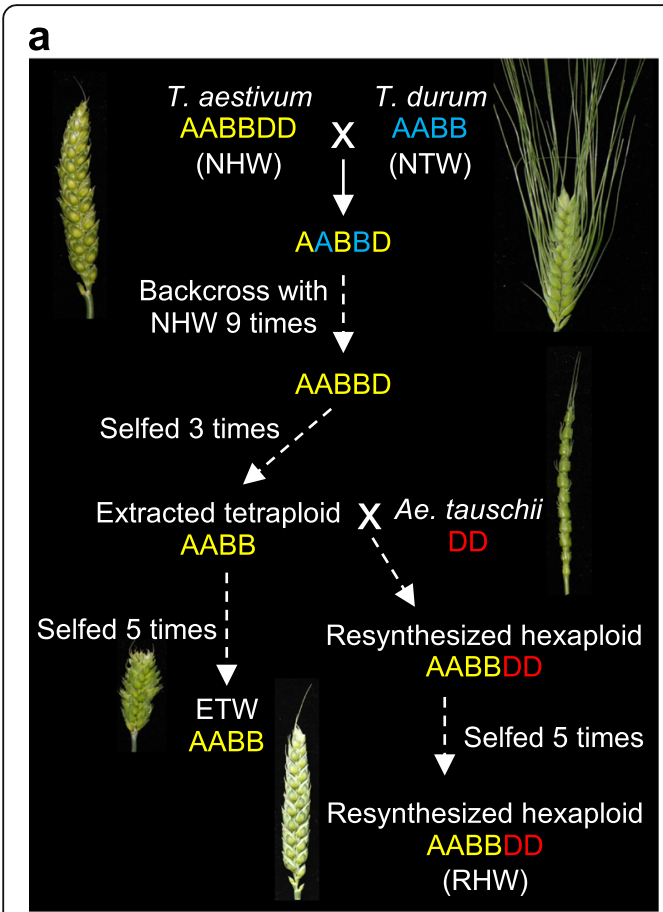

e

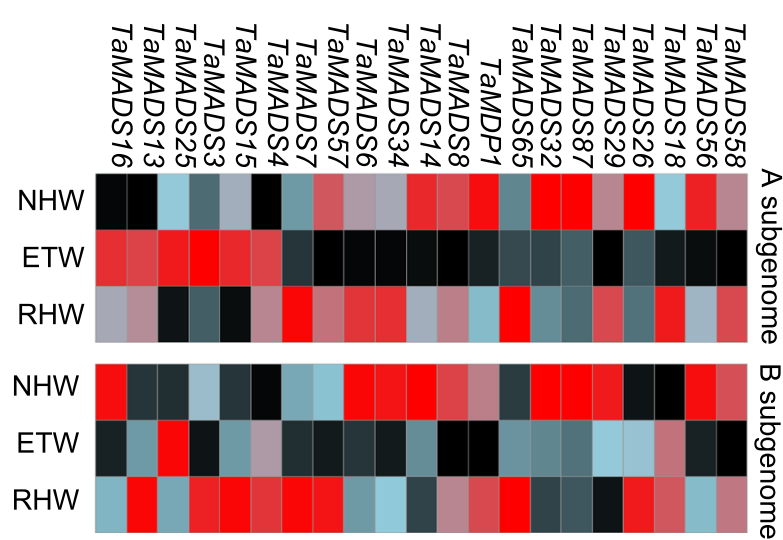

b

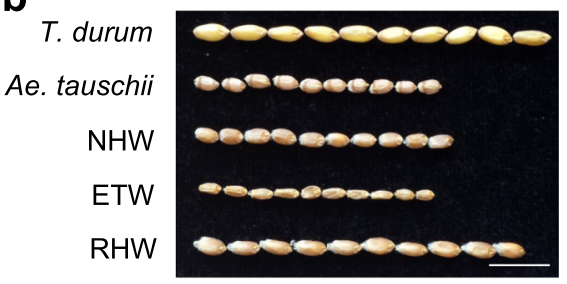

C $\square$ recovered $\llbracket$ unrecovered

d multicellular organismal development pollen-pistil interaction post-embryonic development embryo development catabolic process signal transduction response to stress transport lipid metabolic process cellular protein modification process carbohydrate metabolic process

$P$ value: 0 0.05

Gene number: $\quad \cdot 10 \bullet 100 \bullet 400$

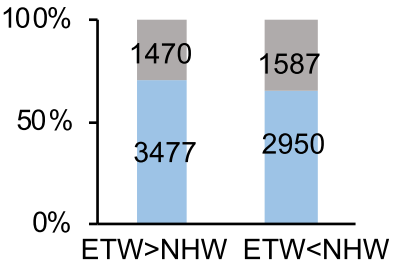

ETW $>$ NHW ETW<NHW

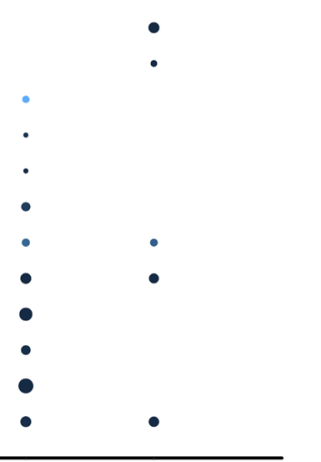

f

\section{NHW ETW RHW NHW ETW RHW}
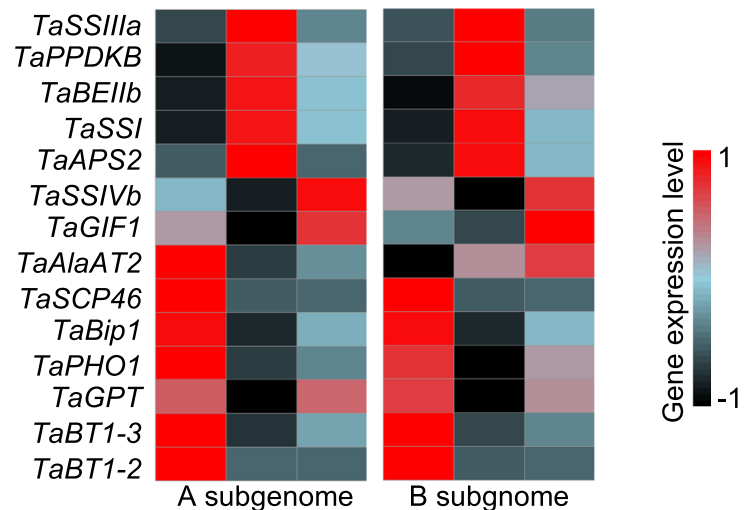

Fig. 1 Differentially expressed genes between extracted tetraploid wheat (ETW, AABB) and natural hexaploid wheat (NHW, AABBDD). a Diagram for producing ETW from NHW, modified from those of $[13,14]$. The hybrid (AABBD) between NHW and NTW was backcrossed with NHW for nine generations, followed by selfing for three generations to select AABB genotype, which was selfed for additonal five generations to maintain genome stability. The resynthesized hexaploid was made between ETW (AABB) and DD and self-pollinated for five generaitons. b Mature seed morphology of T. durum, Ae. tauschii, NHW, ETW, and resynthesized hexaploid wheat (RHW, AABBDD). Scale bar $=1 \mathrm{~cm}$. $\mathbf{c}$ Number of differentially expressed genes (DEGs) between ETW and NHW, with the majority (blue) restored and fewer not restored (yellow) in RHW. d Gene ontology (GO) analysis of DEGs. The circle color and size indicate $P$ value and gene number, respectively (hypergeometric test, $P<0.05$ ). e, $\mathbf{f}$ Heatmaps showing expression levels (fragment per kiobase per million, FPKM) of MADS-box genes (e) and starch biosynthetic genes (f) in ETW, NHW, and RHW

content and small kernel in the ETW (Additional file 1: Figure S1c, d), the genes involved in starch biosynthesis were also downregulated (Fig. 1f).

\section{Genome-wide demethylation in ETW and remethylation after regain of DD in $\mathrm{RHW}$}

Similar to phenotypic and gene expression changes, overall DNA methylation levels of immunofluorescence- stained chromosomes were decreased in the ETW and returned to the same status after gaining the D subgenome in the resynthesized allohexaploid wheat (RHW) as in NHW (Fig. 2a, b). To reveal DNA methylation changes before and after genome separation and merger, we performed whole-genome methylcytosine sequencing (MethylC-seq) in the endosperm of ETW, NHW, RHW, NTW (T. durum), and diploid (Ae. tauschii, DD) lines 


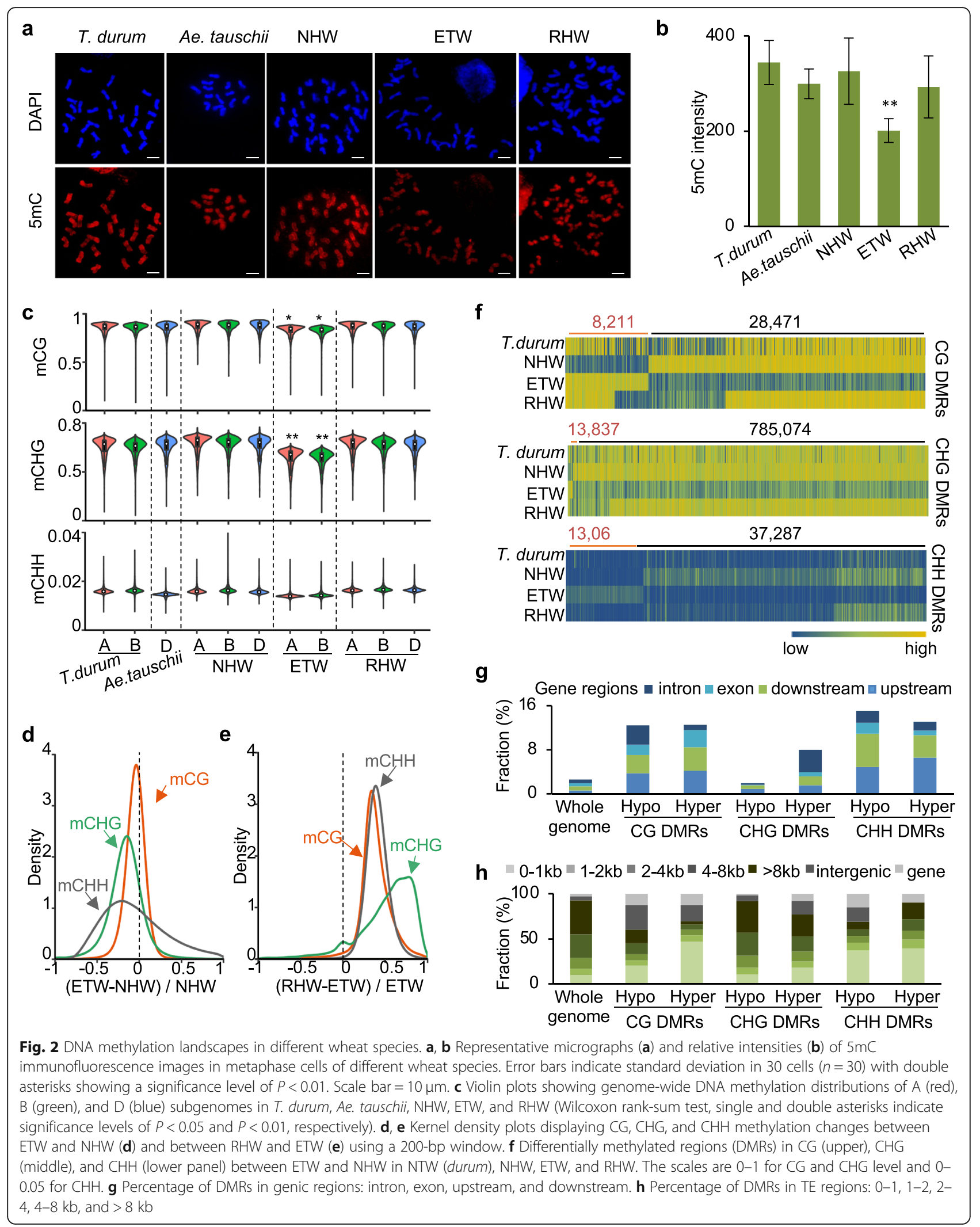


(Additional file 2: Table S3). To avoid potential effects of genomic variation on MethylC-seq analysis, we resequenced T. durum, Ae. tauschii, ETW, and NHW (Additional file 2: Table S4) and identified 28,536,651, 9,059, $622,18,513,346$, and $23,414,348$ SNPs, as well as 1,721 , $347,895,291,1,165,534$, and 1,595,081 insertions/deletions (Indels), respectively, relative to the Chinese Spring genome (WGAv1) [11]. In addition, a total of 25,774 copy number variations (CNVs) were identified in all lines relative to the Chinese Spring genome (WGAv1). MethylC-seq reads were mapped to the SNP-corrected Chinese Spring genome (WGAv1), excluding those in the Indel and CNV regions, for further analysis. The bisulfite-conversion rate was $>99 \%$ (Additional file 2: Table S3), with a mean coverage of cytosines at $\sim 8.8$-fold of genome equivalent for all genotypes (Additional file 2: Table S3). Consistent with decreased methylation levels by immunofluorescence analysis, CG and CHG methylation levels were decreased in both A and B subgenomes of ETW compared to NHW (Fig. 2c-e and Additional file 1: Figure S3a, 3b) (Wilcoxon rank-sum test, $P<0.05$ ), while the methylation levels were recovered in the RHW, to a similar level as in the NHW (Fig. 2c-e).

Further analysis identified predominately hypodifferentially methylated regions (hypo-DMRs) between ETW and NHW in CG $(28,471)$, CHG $(785,074)$, and $\mathrm{CHH}(37,287)$ sites (Fig. 2f), whereas hyper-DMRs were substantially lower in all contexts, including 8211 (CG), 13,837 (CHG), and 13,063 (CHH), respectively (Fig. 2f). Among hypo-DMRs, the majority in CG (22,340, 78.4\%) and CHG $(707,665,90.1 \%)$ plus one-third in $\mathrm{CHH}(12$, $308,33.0 \%$ ) were reverted to higher levels in the RHW (Fig. 2f and Additional file 2: Table S5). All CG and $\mathrm{CHH}$ DMRs, except for CHG hypo-DMRs, were enriched in genic regions (Fig. 2g), suggesting their potential roles in gene expression. For TE regions, CG hyper-DMRs and $\mathrm{CHH}$ DMRs were enriched in short TEs (Fig. 2h). The distribution pattern of $\mathrm{CHG}$ hypo-DMRs was similar to the whole-genome methylation profile in both genic and TE regions (Fig. 2g, h), indicating global demethylation of CHG context throughout two subgenomes in the ETW (Additional file 1: Figure S3b).

In ETW, CG, CHG, and CHH hypo-DMRs overlap with 1520, 9612, and 964 genes in their promoter regions, respectively (Additional file 2: Table S6). These genes are overrepresented in GO terms of metabolism and translation-related processes, including photosynthesis, metabolic process, and translation (Fig. 3a, hypergeometric test, $P<0.05)$. The function of photosynthesis in seed development is unclear. In Arabidopsis, photosynthetic genes are expressed in the peripheral endosperm [20], which may suggest a role for photosynthetic activities in the endosperm and seed coat development.
Notably, most hyper- or hypo-DMRs were associated with only one homoeolog (Fig. 3b), implying a role for DNA methylation in biased expression of homoeologs. The fraction of DMRs associated with both $\mathrm{A}$ and $\mathrm{B}$ homoeologs was relatively small, albeit a slightly higher fraction $(696, \sim 23 \%$ ) of CHG hypo-DMRs with both homoeologs. TaDOS is an example. OsDOS, a rice homolog, is known to regulate spike development and fertility [21]. Both A and B homoeologs of TaDOS were demethylated and expressed at higher levels in the ETW than in RHW and NHW (Fig. 3c, d), suggesting a potential role for TaDOS in seed phenotypic changes in the extracted tetraploid wheat.

\section{Unique genomic regions of demethylation and TE activation in ETW}

Genome-wide hypomethylation could activate TEs in the ETW. Indeed, among 5117 differentially expressed TEs between ETW and RHW (Fig. 4a), 36.3\% (1860) and $12.3 \%$ (628) TEs were expressed specifically in ETW and NHW, respectively (Fig. 4b). The TEs that were activated in ETW were dramatically demethylated, whereas the methylation levels were similarly low in the coding regions among all TEs and NHWexpressed TEs (Fig. 4b). Among ETW-activated TEs, DNA-Harbinger, LTR, and LINE TEs were overrepresented (Fig. 4c, hypergeometric test, $P<0.05$ ). For example, a LTR TE in the ETW was hypomethylated in CG, CHG, and CHH sites and highly expressed (Fig. 4d). These data suggest that TEs are demethylated and activated in ETW, which may be methylated and silenced in the natural hexaploid wheat.

Next, we examined if ETW and NTW (T. durum) share similar methylation profiles since they have similar genomic composition (AABB) and conserved karyotypes (Additional file 1: Figure S2) [13]. There were 229,739 CG, 567,555 CHG, and 64,184 CHH DMRs between T. durum and NHW (Additional file 1: Figure S4a), and the number was not significantly different between hyperand hypo-DMRs. Although these DMRs were evenly distributed between A and B subgenomes (Additional file 1: Figure S4a), only $2 \sim 12 \%$ of DMRs overlapped between ETW and T. durum (Additional file 1: Figure S4b). Moreover, different TEs were expressed specifically in ETW or $T$. durum. These data may suggest that genome-wide demethylation and TE activation in the ETW occur at the genomic regions that are different from those in the natural tetraploid wheat.

Using the resequencing data, we identified subgenomic TEs (see the "Methods" section), including 4933 (A subgenome), 8005 (B subgenome), and 4047 (D subgenome), which were present only in the natural hexaploid wheat but absent in the natural tetraploid wheat or D-genome diploid (Fig. 4e and 


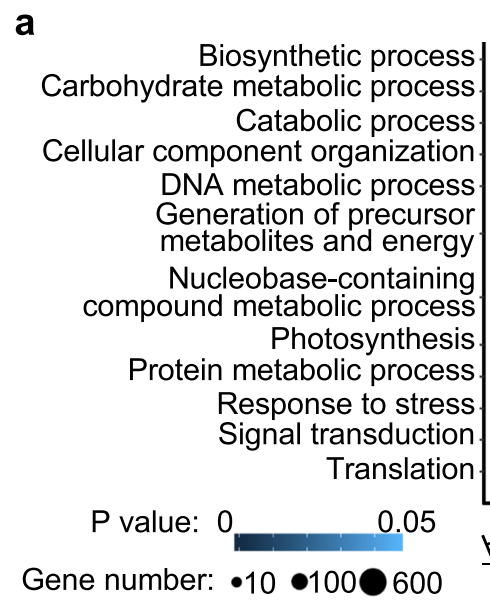

a Biosynthetic process Carbohydrate metabolic process Catabolic process
Cellular component organization DNA metabolic process Generation of precursor metabolites and energy

Nucleobase-containing compound metabolic process Photosynthesis Protein metabolic process Response to stress Signal transduction Translation Gene number: •10 •100 600

b

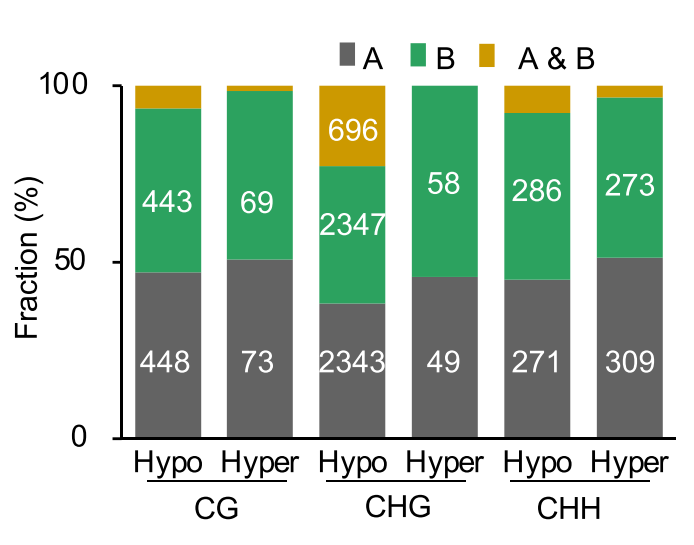

chr3B:

C chr3A: $71,789,752 \sim 71,800,017$

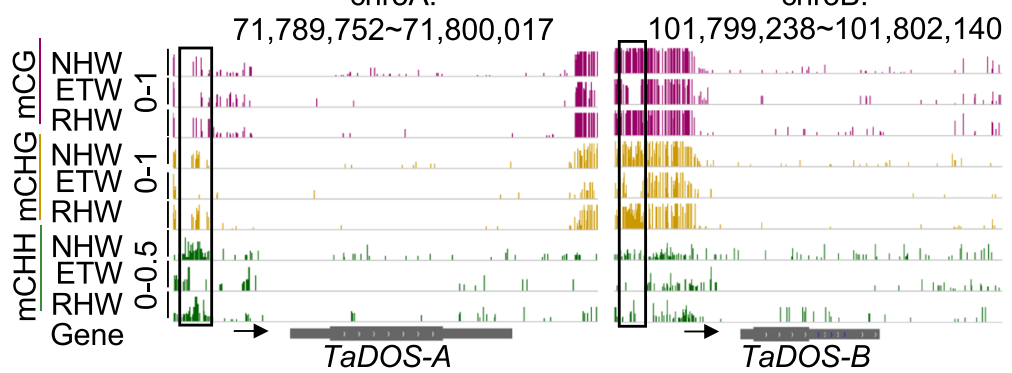

d

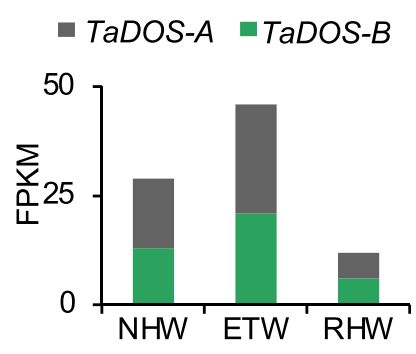

Fig. 3 Relationship between gene expression and DNA methylation in ETW. a GO representation of DMR-associated genes in ETW. The circle color and size indicate $P$ value and gene number, respectively. $\mathbf{b}$ DNA methylation changes in A (gray), B (green), and both A and B (yellow) homoeologous genes in ETW. c An example showing DNA methylation decrease in both homoeologs of TaDOS in ETW. The scales are 0-1 for CG and CHG and 0-0.05 for CHH. d Expression levels (FPKM) of TaDOS homoelogs

Additional file 2: Table S7). These TEs were distributed predominately in chromosome arms and sparsely in pericentromeric regions (Fig. 4e), which were different from distribution patterns of other shared TEs (present in all NHW, NTW, and D-genome diploid lines) (Additional file 1: Figure S3b). Many of these TEs were associated with genes, suggesting that hexaploid bread wheat may have shaped DNA methylation and gene expression diversity for selection, adaptation, and domestication. Two exceptions exist for chromosomes $4 \mathrm{~B}$ and $7 \mathrm{~B}$, where new TEs are present mostly in centromeric regions (Fig. 4e).

These TEs are enriched for DNA transposons, including Harbinger, Mutator, and hAT (Fig. 4f, hypergeometric test, $P<0.05)$. In addition, DNA methylation levels of neighboring regions in these TEs were substantially increased compared to the shared TEs (Fig. 4g, Wilcoxon rank-sum test, $P<0.05$ ). Among them, 1366 (A), 1184 (B), and 466 (D) TEs were present in the upstream (5'), downstream (3'), and the gene-body regions, respectively (Fig. $4 \mathrm{~h}$ ). The fraction of DEGs between the hexaploid wheat and its diploid and tetraploid relatives was significantly higher in the TE regions than that in all genes (Fig. 4i, hypergeometric test,
$P<0.05)$ (DEGs/all expressed genes). These TE-associated genes were overrepresented in cellular protein modification process, nucleotide binding, and carbohydrate binding (Additional file 1: Figure S4c, hypergeometric test, $P<$ 0.05). For example, TraesCS2B01G454000 has a TE in its promoter and is hypermethylated and expressed at low levels (Additional file 1: Figure S4d). These TEs present in genic regions may induce DNA methylation through RNA-directed DNA methylation [22], which in turn suppresses expression of TE-associated genes in the hexaploid wheat.

\section{Decreased H3K9me2 and increased histone expression levels in ETW}

Genome-wide CHG demethylation in ETW may suggest a role for $\mathrm{H} 3 \mathrm{~K} 9 \mathrm{me} 2$, as it is highly correlated with CHG methylation in plants [23]. Indeed, we found that overall H3K9me2 levels were reduced in ETW and reverted to the normal level in RHW (Fig. 5a, b, $t$ test, $P<0.05$ ). The reduced levels of $\mathrm{H} 3 \mathrm{~K} 9 \mathrm{me} 2$ in ETW were associated with the upregulation of $\mathrm{H} 3 \mathrm{~K} 9 \mathrm{me} 2$ demethylase genes such as SDG709 and SDG710 and downregulation of H3K9me2 methyltransferase genes such as JMJ706 (Fig. 5c, d and Additional file 1: Figure S5a). Moreover, 
a

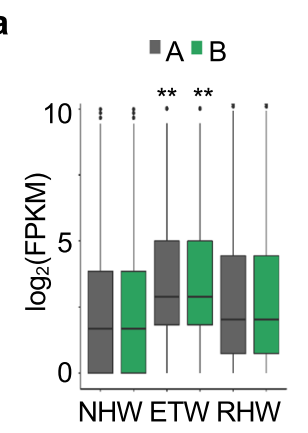

b

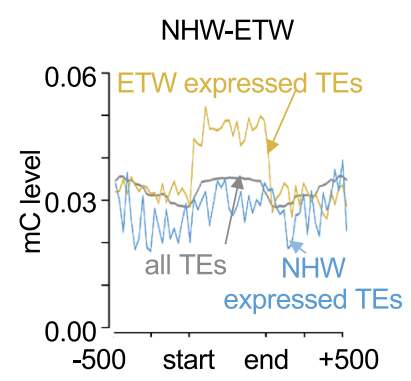

C

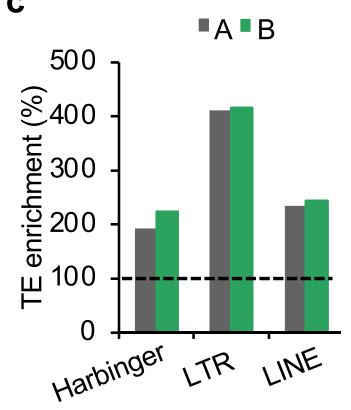

d

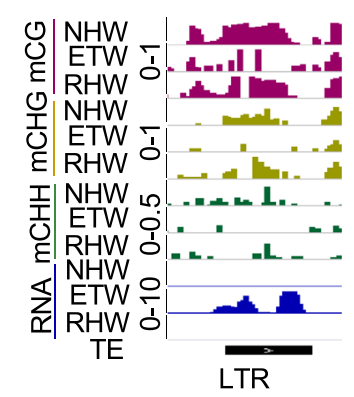

h

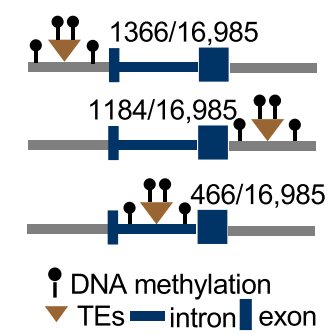

i

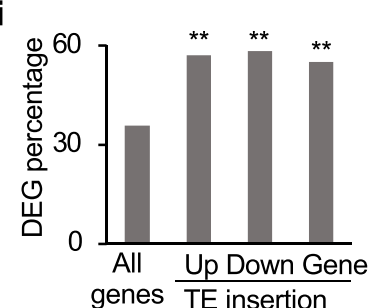

Fig. 4 DNA methylation variation, TE expression, and expansion during wheat polyploidization. a Expression levels of differentially expressed TEs in NHW, ETW, and RHW (Wilcoxon rank-sum test with double asterisks indicating a significant level of $P<0.01$ ). $\mathbf{b}$ Methylation changes of TEs that were expressed specifically in the ETW (yellow line) and NHW (blue line) using all TEs (gray line) as a control. c Overrepresentation of expressed TEs in three TE groups (hypergeometric test, $P<0.05$ ). $\mathbf{d}$ An example of TE with increased expression and reduced DNA methylation levels. The box indicates the DMR region. The scales are $0-1$ for $\mathrm{CG}$ and $\mathrm{CHG}, 0-0.05$ for $\mathrm{CHH}$, and 0-50 for FPKM. e Distribution of TEs in NHW relative to NTW and D-genome diploid in A (red), B (green), and D (blue) subgenomes. f Overrepresentation of five TE groups in A (red), B (green), and D (blue) subgenomes. $\mathbf{g}$ CG methylation density plots showing an increase in the upstream and downstream regions of the TEs in NHW relative to NTW (blue) and D-genome diploid (purple). Shared TEs in NHW and NTW are used as a control. $\mathbf{h}$ Distribution of TEs in upstream, downstream, and gene body. i Percentage of DEGs with TE insertions in upstream, downstream, and gene body using all expressed genes as a control. Double asterisks indicate a significance level of $P<0.01$ (hypergeometric test)

many genes encoding histone $\mathrm{H} 2 \mathrm{~A}, \mathrm{H} 2 \mathrm{~B}$, and $\mathrm{H} 4$ were also upregulated in ETW (Fig. 5e), which correlated with decreased CHG methylation levels in their putative promoter regions. In plants, there are several histone $\mathrm{H} 2$ variants, including H2A.Z, H2A.X, and H2A.W [24]. DNA methylation can influence chromatin structure and affect gene silencing by excluding histone H2A.Z and H2A.Z [25]. We found that the expression of several histone $\mathrm{H} 2$ variants was also upregulated and correlated with DNA methylation changes in their putative promoter regions (Fig. 5e and Additional file 1: Figure S5b). For example, TraesCS2B01G239600, encoding H2A.X, was demethylated at its putative promoter region and expressed at higher levels in ETW than in NHW and RHW (Fig. 5f). These data suggest that overall chromatin modifications including decreased H3K9me2 levels and increased histone variant distribution may contribute to demethylation in ETW after genome separation from natural hexaploid wheat.

\section{Discussion}

Hexaploid wheat was formed $8000-10,000$ years ago by hybridizing a tetraploid wheat with a D-genome diploid coupled with genome doubling [8]. After polyploidization, three sets of subgenomes (A, B, and D) could have experienced genetic and epigenetic modifications. In newly formed allotetraploid wheat, sequence elimination and DNA methylation can occur rapidly [26], which lead to expression bias among homoeologs [27]. Estimates indicate that 30\% homoeologous triads in hexaploid bread wheat display 


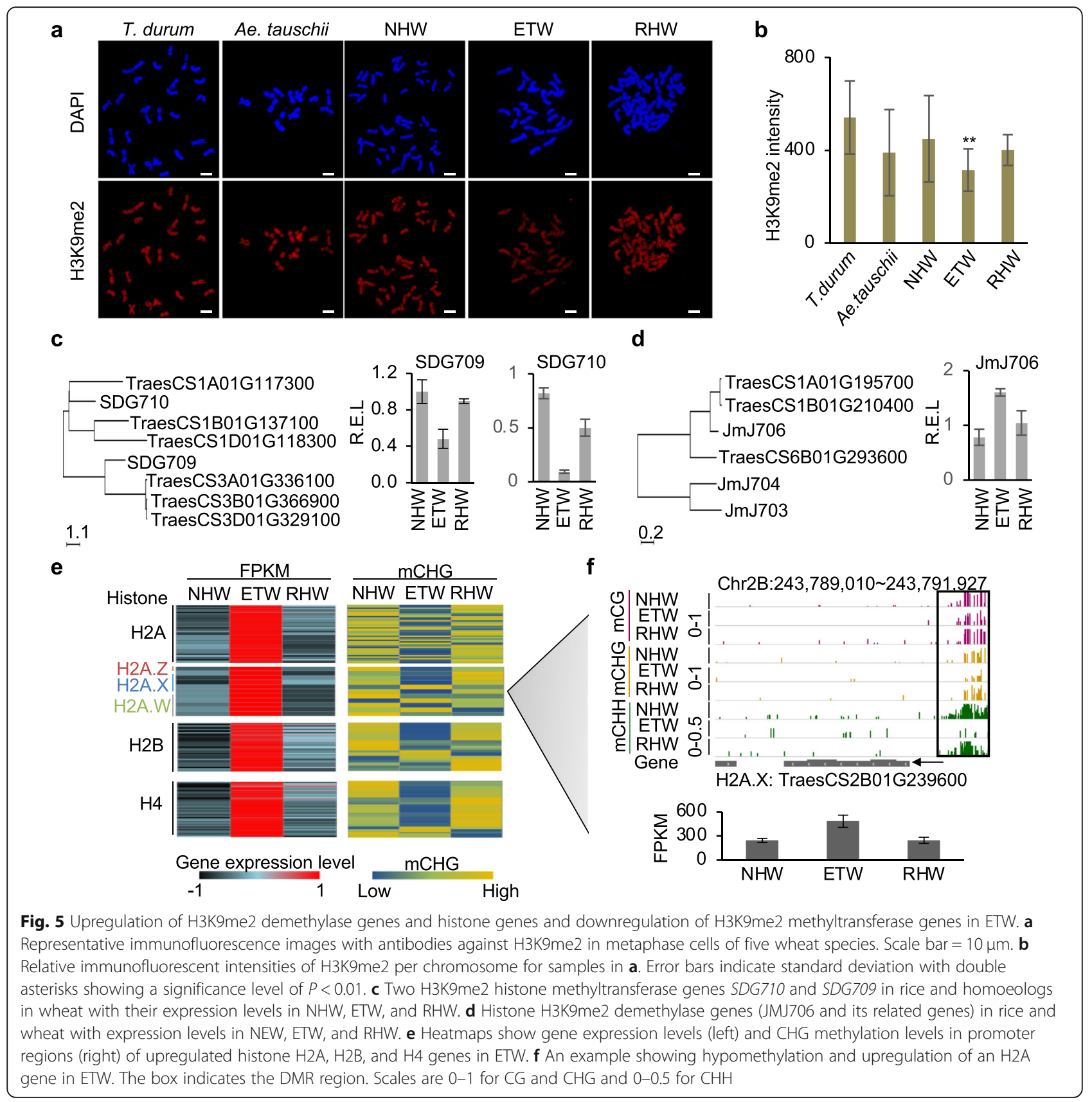

biased expression [28], and some genes with the expression bias are associated with DNA methylation and TE variation within promoter regions of those homoeologs [29]. Notably, homoeologous chromosomes in the ETW (AABB) pair normally during meiosis and show little genetic variation (Additional file 1: Figure S2) [13]. This may suggest more epigenetic changes than genetic changes in the ETW after genome separation, as previously reported [15]. It is likely that epigenetic modifications have been reprogrammed after the formation of hexaploid wheat and during 10,000 years of evolution and domestication. One such modification is DNA methylation, which has been reduced in ETW, compared to NHW and NTW. This global hypomethylation is dependent on the trans-effect by the D subgenome. When a D-genome diploid is merged with the ETW to form a hexaploid wheat, the methylation levels return to the normal level. This remethylation at the genome-wide level is unlikely from spontaneous mutants during separation and merger of the D genome in hexaploid wheat. Interspecific hybridization may induce DNA methylation changes that are stably maintained in the hexaploid wheat, as observed in five allotetraploid cotton species [4, 30, 31]. Further studies using additional sets of extracted and resynthesized wheat and/or other polyploid plants should address if this 
demethylation-remethylation is a rule or exception for the genome separation and merger during polyploidization.

Genome-wide demethylation occurs in the endosperm, as observed in Arabidopsis [32, 33] and rice [34, 35]. In wheat, this demethylation in the endosperm is more profound in the ETW than in the NTW and can be reversed after the addition of the D genome, suggesting a potential effect of the $\mathrm{D}$ genome on remethylation in the hexaploid wheat. However, we cannot rule out other possibilities such as imprinting and activation of other factors in the resynthesized hexaploid wheat, which may also mediate methylation changes in the endosperm. In addition, it will be interesting to test if this type of methylation changes also occurs in other tissues and organs.

Notably, genome-wide DNA methylation reprogramming that occurred in the ETW (AABB separated from the hexaploid, AABBDD) is different from the overall methylation distribution in natural tetraploid wheat (AABB). Only 2 12\% DMRs (relative to NHW) overlap between ETW and T. durum. This suggests that interactions between $A B$ and $D$ subgenomes during interspecific hybridization and polyploidization could induce epigenetic changes in genomic regions that are different from those present in the progenitor-like AABB genomes. Alternatively, the extant tetraploid wheat ( $T$. durum) may not be the tetraploid donor of hexaploid wheat, because the exact progenitor-like tetraploid and diploid species are unknown [8] or became extinct. Interestingly, the DNA methylation changes in the ETW after separation from the D subgenome could be restored by the addition of the $\mathrm{D}$ genome. This may indicate inter-dependency of epigenetic modifications between three subgenomes in the allohexaploid wheat. The reduced DNA methylation in the ETW (AABB) after separation of DD is similar to the phenomenon of trans-acting demethylation (TAdM), as observed in Arabidopsis [36]. These trans-acting factors could be small RNAs [7].

TEs can represent $\sim 40 \%$ of genome in humans and over $80 \%$ in plants including wheat [11]. Although TEs can determine genome size, generate mutations and chromosome rearrangements, and regulate gene expression, the majority of them are methylated and remain in heterochromatic regions [37]. Global demethylation, especially in CHG hypomethylation, contributes to the activation of TEs in ETW, which can affect genome stability, gene expression, and phenotypic changes. Loss of CHG methylation in the rice oscmt3a mutant induces activation of TEs including Copia and Gypsy elements [38]. In Ae. tauschii, TEs are associated with about half of the genes, and such genes are hypermethylated and expressed at lower levels than those without TEs [39]. Although the genic regions are conserved among three subgenomes in hexaploid wheat [11], there is a massive turnover of TEs since A, B, and D diverged [40]. Using the resequencing data, we found TE distributions more in the B subgenome than A and D subgenomes, consistent with the subgenome size, where the $B$ subgenome is nearly twice the size of A and D subgenomes [11]. Interestingly, these TEs may regulate DNA methylation and expression of TE-associated genes during the evolution and domestication of hexaploid bread wheat.

In plants, H3K9me2 marks are associated with CHG methylation through CMT3-mediated pathway [23], and mutation in SDG714 encoding a H3K9 methyltransferase leads to decreased H3K9 methylation as well as CG and CHG methylation in rice [41]. Consistent with this finding, in ETW where CHG methylation is reduced, H3K9me2 demethylase genes are upregulated, while H3K9me2 methyltransferase genes are downregulated in ETW. As a result, overall H3K9me2 levels on homoeologous chromosomes are generally decreased in ETW. Although the cause and effect remain to be tested, these results indicate concerted epigenetic changes between H3K9me2 and DNA demethylation in ETW. Consistent with this notion, other chromatin factors such as core histone $\mathrm{H} 2 \mathrm{~A}, \mathrm{H} 2 \mathrm{~B}$ and $\mathrm{H} 4$, and histone $\mathrm{H} 2 \mathrm{~A}$ variant genes are hypomethylated in their promoter regions and upregulated in the ETW. Together, these data suggest that intergenomic interactions during genome merger and separation may induce chromatin and DNA methylation changes that are stably maintained in allopolyploids. These concerted epigenetic changes could shape gene expression diversity, TE expansion, and genome stability, which may contribute to the evolution and domestication of polyploid wheat and other crops.

\section{Conclusions}

Genome separation and merger in hexaploid bread wheat can provoke dynamic and reversible changes in DNA methylation and histone modifications, suggesting pervasive epigenetic changes induced in allopolyploids such as wheat and cotton. These changes accompany altered TE activity and gene expression, which may lead to phenotypic variation for selection, adaptation, and domestication. Our results provide unique insights into epigenetic roles in genome merger, separation, and evolution of polyploid wheat and other crops.

\section{Methods}

\section{Plant materials and DNA/RNA extraction}

Plant materials included extracted tetraploid wheat (ETW, AABB), natural hexaploid bread wheat (NHW, $T$. aestivum L. cv Canthach, AABBDD), natural tetraploid wheat (NTW, T. turgidum L. subsp. durum, AABB), Ae. tauschii subsp. strangulata (line RL5288, DD), and resynthesized hexaploid wheat (RHW, AABBDD). Starch content was analyzed with three biological replications 
from 20 seeds at $6,9,12$, and 15 days after pollination (DAP) and maturing stage using the protocol described in the Total Starch Assay Kit (K-TSTA) (Megazyme, Co. Wicklow, Ireland). Endosperm at 6 DAP was manually dissected and used for DNA and RNA extraction [42]. Trefoil stage leaves of T. durum, Ae. tauschii, ETW, and NHW were used for DNA extraction and resequencing. Genomic resequencing, whole-genome bisulfite sequencing, and RNA-seq with three biological replications were performed following the published methods [4].

\section{Analysis of karyotypes, DNA methylation, and H3K9me2 immunofluorescence}

The protocol for fluorescence in situ hybridization (FISH) was adopted as previously described [43]. For FISH, repetitive DNA sequences $p S c 119.2$ and a microsatellite (GAA)n oligonucleotide were labeled with Chroma Tide Alexa Fluor 488-5-dUTP (C11397, Invitrogen, Eugene, USA), and $p A s 1$ was labeled with Texas Red-5-dCTP (NEL 426, Perkin-Elmer, Boston, USA). Anti-methylcytosine antibodies (1982501, Millipore, Temecula, USA) and anti-dimethyl-Histone H3 (Lys9) antibodies (07-441, Millipore, Temecula, USA) were used for immunofluorescence analysis using published protocols [5, 42]. Slides were examined with an Olympus BX61 fluorescence microscope and digitally photographed. Immunofluorescence values of DNA methylation and H3K9me2 were estimated from homoeologous chromosomes of 30 cells using an Olympus cellSens Dimension and were averaged by the number of chromosomes in a given genotype.

\section{Resequencing and identification of SNPs, Indels, CNVs, and TEs}

Sequencing reads (150-bp paired-end) of T. durum, Ae. tauschii, ETW, and NHW were trimmed using NGSQ CToolkit_v2.3 to remove low-quality reads [44]. Highquality reads were mapped onto the genome of $T$. aestivum (Chinese Spring, WGAv1) [11] using BWA (BWAMEM algorithm) with default parameters [45]. Read pairs with a mapping quality lower than 10 were removed. Single nucleotide polymorphisms (SNPs) and insertion-deletions (Indels, 2-50 bp) were identified using samtools, with quality scores $>100$, depth $>10$, and $90 \%$ or more read coverage. SNPs were then substituted in the corresponding reference genomes of each genotype for further analysis. ETW combined with the Ae. tauschii sequence for the analysis of RHW. Copy number variants (CNVs) (deletions and duplications) were analyzed using delly [46] and lumpy [47], each with 5 or more supporting paired-end reads and MAPQ $>20$. CNVs detected by delly and lumpy methods were combined into one file using the loci with $80 \%$ reciprocal overlaps.
The TEs present in hexaploid wheat but not in Ae. tauschii and T. durum were identified using the published methods [48]. We mapped the reads of $A e$. tauschii and T. durum onto $\mathrm{D}$ and $\mathrm{AB}$ subgenomes of $T$. aestivum (Chinese Spring, WGAv1), respectively. Split and discordant reads were extracted to identify absence variants of TEs. A split read was defined to contain the separation point, with the segment separated by more than 2-kb distance. Discordant reads were defined as mate-paired reads that were aligned to the reference genome with more than 2-kb distance. We first identified annotated TEs in the reference genome that were spanned by split and discordant reads. Among these TEs, we then identified the candidates of TE absence variants using the criteria of a split or discordant read spanning at least $80 \%$ of the TE sequence, and the sequencing depth within the spanned region is $<10 \%$ of the 2-kb flanking sequence. In addition, we extracted NHW resequencing reads that were not aligned to the genomes of Ae. tauschii and T. durum and mapped these reads to the genome of $T$. aestivum. Finally, the candidates of TE absence variants with $80 \%$ or more regions covered by resequencing reads of NHW were identified as absence variants of TEs in Ae. tauschii and T. durum relative to hexaploid wheat.

\section{RNA-seq and gene expression analysis}

After removing low-quality reads, RNA-seq reads were aligned with default parameters using TopHat [49] to the corresponding references of each genotype, retaining the uniquely mapped reads with both pairs mapped onto the same chromosome. For interlibrary comparison, RNA-seq reads were normalized to fragment per kiobase per million (FPKM) using Cufflinks [49], and the genes with FPKM $>1$ in at least two biological replications were used for further analysis. The reads from two compared groups were normalized by their respective size factors, which were analyzed by DESeq package (differential expression analysis for sequence count data) (parameter: estimateSizeFactors). Fold-cut and FDR values were 2 and FDR $<0.05$ for differentially expressed genes (DEGs) and 1.5-fold and FDR $<0.05$ for differentially expressed TEs.

Gene Ontology (GO) classification was performed using BLAST software, and GO enrichment analysis was implemented by Blast2go [50]. GO term analysis was performed using GOSlim using all genes in each subgenome or all subgenomes as a control. Hypergeometric test was used for statistical significance tests $(P<0.05)$.

\section{Identification of homoeologs among A, B, and D subgenomes}

Homoeologous genes between each pair of A, B, and D subgenomes in Chinese Spring [11] were identified as 
previously described [42]. Briefly, coding sequences (CDSs) of the two subgenomes were aligned reciprocally with a BLASTN cutoff value of $e^{-10}$ [51]. Gene pairs with over $90 \%$ sequence identity and an overall length of orthologous regions larger than $60 \%$ of the CDSs in both subgenomes were defined as homoeologous genes. The one-to-one reciprocal homoeologs were regarded as dyad. Dyads present in all three comparisons were considered as triad.

\section{Whole-genome DNA methylation analysis}

Bisulfite sequencing reads of NHW, ETW, and RHW were mapped to SNP-corrected Chinese Spring genome sequence (WGAv1). Reads of Ae. tauschii and T. durum were mapped to corresponding SNP-corrected genome sequences, respectively. Read mapping was performed using Bismark (parameter: bowtie2 -N 0) [52]. SNPs in the regions with $\mathrm{CNVs}$ and Indels were excluded from the analysis. Only the cytosines covered by at least three reads in all compared wheat materials were used for further analysis. Cytosines with $P$ value below $10^{-5}$ were identified as methylcytosines (binomial distribution). Genome-wide DNA methylation was calculated using the proportion of methylcytosines in all reads covered cytosines in every chromosome window $(1 \mathrm{Mb})$ and shown using circos plots (http://circos.ca/).

Differentially methylated regions (DMRs) between two genotypes were identified using a published workflow by 200-bp sliding-window [4]. The methylation level was calculated by $(\mathrm{mC}) /(\mathrm{mC}+$ non- $\mathrm{mC})$, in which $\mathrm{mC}$ and non-mC refer to the number of methylated and unmethylated cytosines in one region. The mean methylation level was estimated for each window. A window with both two materials containing 16, 16, and 64 or more reads in CG, CHG, and $\mathrm{CHH}$ content was kept for further analysis. Statistical significance for DMRs in each window was weighted using Fisher's exact test with corrected $P_{\text {adj }}<0.05$. Finally, all significant windows were filtered by the following criteria: the methylation level difference between two alleles was higher than 0.5 (50\%), $0.3(30 \%)$, and $0.1(10 \%)$ in CG, CHG, and $\mathrm{CHH}$ contexts, respectively.

We used a sliding-window approach, dividing the region into 60 windows to show DNA methylation level changes, to display distribution of DNA methylation levels in the gene body with 2000-bp up- and downstream regions and TEs with 500-bp up- and downstream regions. Integrative Genomics Viewer (IGV) [53] was used to show the distribution of DNA methylation and gene expression levels for specific examples.

\section{Quantitative RT-PCR analysis}

After DNase treatment, total RNA $(1 \mu \mathrm{g})$ was reversetranscribed to cDNA using HiScript II Q RT SuperMix for qPCR (Vazyme R223-01). The cDNA was used as the template for qRT-PCR using AceQ qPCR SYBR Green Master Mix (Q111-02, Vazyme, Nanjing, China). A housekeeping gene encoding glyceraldehyde3-phosphate dehydrogenase $(G A D P H)$ was used as a control for expression analysis in Triticeae [14]. Primer pairs for qRTPCR analysis are listed in (Additional file 2: Table S8).

\section{Supplementary Information}

Supplementary information accompanies this paper at https://doi.org/10 1186/s12915-020-00909-x.

Additional file 1: Figure S1. Extracted tetraploid wheat (ETW) shows decreased starch content, smaller starch granule, and reduced fertility. (a) Images showing pollen fertility by iodine staining (top panel) and starch structure by scanning electron microscopy (bottom panel). S refers to starch granules. Materials are T. durum (AABB), Ae. Tauschii (DD), natural hexaploid wheat (NHW, AABBDD), ETW (AABB), and resynthesized hexaploid wheat (RHW, AABBDD). Scale bars $=100 \mu \mathrm{m}$ (top images) and $15 \mu \mathrm{m}$ (bottom images). (b) Developing seed morphology at 6 days after pollination (DAP) in the same set of lines as in (a). Scale bar $=0.5 \mathrm{~cm}$ for all images. (c, d) Total starch content ( $\mathrm{mg} / 100 \mathrm{mg}$ ) (c) and thousandkernel weight (grams) (d) of the same set of lines as in (a). Error bars indicate standard deviation of three biological replicates with three asterisk showing a statistical significance level of $P<0.0001$. Figure S2. Karyotypes of five wheat species. Karyotypes of Ae. tauschii (DD), T. durum (AABB), ETW, NHW and RHW. The probes used in FISH are pSc119.2 (green), pAs1 (red) and (GAA) n (yellow). Scale bar $=10 \mu \mathrm{m}$. Figure S3. The DNA methylation levels between ETW (AABB) and NHW (AABBDD). (a) Fraction of methylated cytosine (mC) in T. durum (blue), Ae. tauschii (purple), NHW (green), ETW (yellow) and RHW (pink). One asterisk indicates a statistical significance level of $P<0.05$. (b) Circos plot showing CG, $\mathrm{CHG}$ and $\mathrm{CHH}$ methylation levels in the B subgenome of NHW, ETW and RHW. Blue and gray circos indicate gene and TE densities, respectively. Scales are 0-1 for $\mathrm{CG}$ and $\mathrm{CHG}, 0-0.05$ for $\mathrm{CHH}$. Figure S4. DNA methylation variation and TE expansion during wheat polyploidization. (a) DMRs of A, B or D subgenome between T. durum, Ae. tauschii (DD) and NHW (AABBDD). Scales are 0-1 for $\mathrm{CG}$ and $\mathrm{CHG}, 0-0.05$ for $\mathrm{CH}$. (b) Comparative analysis of DMRs between ETW and NHW with those between $T$. durum and NHW. (c) Gene ontology (GO) representation of the genes with the TEs inserted in the A (red), B (green) or D (blue) subgenome. CPMP: cellular protein modification process; NB: nucleotide binding; KA: kinase activity; TA: transferase activity; CB: carbohydrate binding. Hypergeometric test $(P<0.05)$ was used for statistical significance analysis. $(d)$ An example of the TE-associated gene that shows expression changes. The box region indicates the region of TE insertion and DNA methylation changes. Scales are 0-1 for $\mathrm{CG}$ and $\mathrm{CHG}, 0-0.5$ for $\mathrm{CHH}$. (e) The TE was present in NHW (top) and related to expression changes (fragments per kilobase pairs per million, RPKM) in the TE-associated gene (bottom).

Figure S5. Expression and phylogenetic analyses of DNA and histone methylation-related genes and H2A families in ETW and NHW. (a) Expression levels (logRPKM) of putative DNA methylation-related genes (MET1b, CMT3b, DDM1a, CMT2, ROS1, and DNG703) and H3K9me2 writers (SDG701, SDG703, SDG704, SDG710, SDG723, and SDG726) and erasers (JmJ706) (b) Phylogenetic relationships of $\mathrm{H} 2 \mathrm{~A}$ gene families in hexaploidy wheat.

Additional file 2. Supplementary Tables.

\section{Acknowledgements}

We thank Professor Yufeng Wu and the Bioinformatics Center at Nanjing Agricultural University for the assistance in the data analysis. We are grateful to Dr. E.R. Kerber and Dr. M. Feldman for providing the initial seeds of all plants used in this study.

Authors' contributions

J.Y., W.J., and Z.J.C. designed the research. J.Y., W.J., and Y.L. performed experiments. W.Y., X.W., and B.L. provided reagents and analytical tools. J.Y., 
W.J., Y.L., Q.S., and Z.J.C. analyzed the data. J.Y., Q.S., and Z.J.C. wrote the paper. All authors read and approved the final manuscript.

\section{Funding}

Funding was provided by the National Natural Science Foundation of China (31801351 and 31290213) and the National Key Research and Development Program (2019/KJQN201907). Z.J.C. is the D. J. Sibley Centennial Professor of Plant Molecular Genetics.

\section{Availability of data and materials}

Sequencing data are deposited under accession number CRA002143 in Genome Sequence Archive in BIG Data Center https://bigd.big.ac.cn/gsa [54] The use of plant materials follows local, national, or international guidelines and legislation and the required or appropriate permissions and/or licenses for the study.

\section{Competing interests}

The authors declare no competing interests in this work.

\section{Author details}

${ }^{1}$ State Key Laboratory of Crop Genetics and Germplasm Enhancement, Nanjing Agricultural University, 1 Weigang Road, Nanjing 210095, China. ${ }^{2}$ Key Laboratory of Molecular Epigenetics of the Ministry of Education, Northeast Normal University, Changchu 130024, China. ${ }^{3}$ Department of Molecular Biosciences, The University of Texas at Austin, Austin, TX 78712, USA.

Received: 25 July 2020 Accepted: 28 October 2020

Published online: 20 November 2020

\section{References}

1. Wendel JF, Jackson SA, Meyers BC, Wing RA. Evolution of plant genome architecture. Genome Biol. 2016;17:37.

2. Soltis DE, Visger CJ, Soltis PS. The polyploidy revolution then...and now: Stebbins revisited. Am J Bot. 2014;101:1057-78.

3. Van de Peer Y, Mizrachi E, Marchal K. The evolutionary significance of polyploidy. Nat Rev Genet. 2017;18:411-24.

4. Song Q, Zhang T, Stelly DM, Chen ZJ. Epigenomic and functional analyses reveal roles of epialleles in the loss of photoperiod sensitivity during domestication of allotetraploid cottons. Genome Biol. 2017;18:99.

5. Zheng D, Ye W, Song Q, Han F, Zhang T, Chen ZJ. Histone modifications define expression bias of homoeologous genomes in allotetraploid cotton. Plant Physiol. 2016;172:1760-71.

6. Zhao T, Tao X, Feng S, Wang L, Hong H, Ma W, Shang G, Guo S, He Y, Zhou B, Guan X. LncRNAs in polyploid cotton interspecific hybrids are derived from transposon neofunctionalization. Genome Biol. 2018;19:195.

7. Ha M, Lu J, Tian L, Ramachandran V, Kasschau KD, Chapman EJ, Carrington JC, Chen X, Wang XJ, Chen ZJ. Small RNAs serve as a genetic buffer against genomic shock in Arabidopsis interspecific hybrids and allopolyploids. Proc Natl Acad Sci U S A. 2009;106:17835-40.

8. Salamini F, Ozkan H, Brandolini A, Schafer-Pregl R, Martin W. Genetics and geography of wild cereal domestication in the near east. Nat Rev Genet. 2002;3:429-41.

9. Bevan MW, Uauy C, Wulff BB, Zhou J, Krasileva K, Clark MD. Genomic innovation for crop improvement. Nature. 2017:543:346-54.

10. Griffiths S, Sharp R, Foote TN, Bertin I, Wanous M, Reader S, Colas I, Moore G. Molecular characterization of Ph1 as a major chromosome pairing locus in polyploid wheat. Nature. 2006;439:749-52.

11. International Wheat Genome Sequencing $C$, investigators IRp, Appels R, Eversole K, Feuillet C, Keller B, Rogers J, Stein N, investigators Iw-gap, Pozniak CJ, et al: Shifting the limits in wheat research and breeding using a fully annotated reference genome. Science 2018;361:eaar7197.

12. Brenchley R, Spannagl M, Pfeifer M, Barker GL, D'Amore R, Allen AM, McKenzie N, Kramer M, Kerhornou A, Bolser D, et al. Analysis of the bread wheat genome using whole-genome shotgun sequencing. Nature. 2012; 491:705-10.

13. Kerber ER. Wheat: reconstitution of the tetraploid component (AABB) of hexaploids. Science. 1964;143:253-5.

14. Zhang H, Zhu B, Qi B, Gou X, Dong Y, Xu C, Zhang B, Huang W, Liu C, Wang $X$, et al. Evolution of the BBAA component of bread wheat during its history at the allohexaploid level. Plant Cell. 2014;27:2761-76.
15. Liu C, Yang X, Zhang H, Wang X, Zhang Z, Bian Y, Zhu B, Dong Y, Liu B. Genetic and epigenetic modifications to the BBAA component of common wheat during its evolutionary history at the hexaploid level. Plant Mol Biol. 2015:88:53-64.

16. Gillies SA, Futardo A, Henry RJ. Gene expression in the developing aleurone and starchy endosperm of wheat. Plant Biotechnol J. 2012;10:668-79.

17. Bemer M, Heijmans K, Airoldi C, Davies B, Angenent GC. An atlas of type I MADS box gene expression during female gametophyte and seed development in Arabidopsis. Plant Physiol. 2010;154:287-300.

18. Yin $L L$, Xue HW. The MADS29 transcription factor regulates the degradation of the nucellus and the nucellar projection during rice seed development Plant Cell. 2012;24:1049-65.

19. Cui R, Han J, Zhao S, Su K, Wu F, Du X, Xu Q, Chong K, Theissen G, Meng Z. Functional conservation and diversification of class $E$ floral homeotic genes in rice (Oryza sativa). Plant J. 2010;61:767-81.

20. Belmonte MF, Kirkbride RC, Stone SL, Pelletier JM, Bui AQ, Yeung EC, Hashimoto M, Fei J, Harada CM, Munoz MD, et al. Comprehensive developmental profiles of gene activity in regions and subregions of the Arabidopsis seed. Proc Natl Acad Sci U S A. 2013;110:E435-44.

21. Kong Z, Li M, Yang W, Xu W, Xue Y. A novel nuclear-localized CCCH-type zinc finger protein, OsDOS, is involved in delaying leaf senescence in rice. Plant Physiol. 2006;141:1376-88.

22. Matzke MA, Mosher RA. RNA-directed DNA methylation: an epigenetic pathway of increasing complexity. Nat Rev Genet. 2014;15:394-408.

23. Law JA, Jacobsen SE. Establishing, maintaining and modifying DNA methylation patterns in plants and animals. Nat Rev Genet. 2010;11:204-20.

24. Redon C, Pilch D, Rogakou E, Sedelnikova O, Newrock K, Bonner W. Histone H2A variants H2AX and H2AZ. Curr Opin Genet Dev. 2002;12:162-9.

25. Zilberman D, Coleman-Derr D, Ballinger T, Henikoff S. Histone H2A.Z and DNA methylation are mutually antagonistic chromatin marks. Nature. 2008; 456:125-9.

26. Shaked H, Kashkush K, Ozkan H, Feldman M, Levy AA. Sequence elimination and cytosine methylation are rapid and reproducible responses of the genome to wide hybridization and allopolyploidy in wheat. Plant Cell. 2001; 13:1749-59.

27. Wang X, Zhang H, Li Y, Zhang Z, Li L, Liu B. Transcriptome asymmetry in synthetic and natural allotetraploid wheats, revealed by RNA-sequencing. New Phytol. 2016;209:1264-77.

28. Ramirez-Gonzalez RH, Borrill P, Lang D, Harrington SA, Brinton J, Venturini L, Davey $\mathrm{M}$, Jacobs J, van Ex F, Pasha $A$, et al. The transcriptional landscape of polyploid wheat. Science. 2018;361:eaar6089.

29. Gardiner $\sqcup$, Quinton-Tulloch M, Olohan L, Price J, Hall N, Hall A. A genome-wide survey of DNA methylation in hexaploid wheat. Genome Biol. 2015;16:273.

30. Chen ZJ, Sreedasyam A, Ando A, Song Q, De Santiago LM, Hulse-Kemp AM, Ding M, Ye W, Kirkbride RC, Jenkins J, et al. Genomic diversifications of five Gossypium allopolyploid species and their impact on cotton improvement. Nat Genet. 2020;52:525-33.

31. Ding M, Chen ZJ. Epigenetic perspectives on the evolution and domestication of polyploid plants and crops. Curr Opin Plant Biol. 2018;42: 37-48.

32. Hsieh TF, Ibarra CA, Silva P, Zemach A, Eshed-Williams L, Fischer RL, Zilberman D. Genome-wide demethylation of Arabidopsis endosperm. Science. 2009;324:1451-4

33. Gehring M, Bubb KL, Henikoff S. Extensive demethylation of repetitive elements during seed development underlies gene imprinting. Science. 2009;324:1447-51.

34. Zemach A, Kim MY, Silva P, Rodrigues JA, Dotson B, Brooks MD, Zilberman D. Local DNA hypomethylation activates genes in rice endosperm. Proc Natl Acad Sci U S A. 2010:107:18729-34.

35. Yuan J, Chen S, Jiao W, Wang L, Wang L, Ye W, Lu J, Hong D, You S, Cheng $Z$, et al. Both maternally and paternally imprinted genes regulate seed development in rice. New Phytol. 2017;216:373-87.

36. Greaves IK, Groszmann M, Ying H, Taylor JM, Peacock WJ, Dennis ES. Trans chromosomal methylation in Arabidopsis hybrids. Proc Natl Acad Sci U S A. 2012;109:3570-5.

37. Feschotte C, Jiang N, Wessler SR. Plant transposable elements: where genetics meets genomics. Nat Rev Genet. 2002;3:329-41.

38. Cheng C, Tarutani Y, Miyao A, Ito T, Yamazaki M, Sakai H, Fukai E, Hirochika $\mathrm{H}$. Loss of function mutations in the rice chromomethylase OsCMT3a cause a burst of transposition. Plant J. 2015:83:1069-81. 
39. Zhao G, Zou C, Li K, Wang K, Li T, Gao L, Zhang X, Wang H, Yang Z, Liu X, et al. The Aegilops tauschii genome reveals multiple impacts of transposons. Nat Plants. 2017;3:946-55.

40. Wicker T, Gundlach H, Spannagl M, Uauy C, Borrill P, Ramirez-Gonzalez RH, De Oliveira R, International Wheat Genome Sequencing C, Mayer KFX, Paux

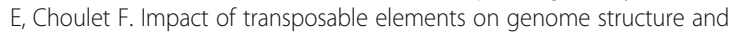
evolution in bread wheat. Genome Biol. 2018;19:103.

41. Ding Y, Wang X, Su L, Zhai J, Cao S, Zhang D, Liu C, Bi Y, Qian Q, Cheng Z, et al. SDG714, a histone H3K9 methyltransferase, is involved in Tos17 DNA methylation and transposition in rice. Plant Cell. 2007;19:9-22.

42. Jiao W, Yuan J, Jiang S, Liu Y, Wang L, Liu M, Zheng D, Ye W, Wang X, Chen ZJ. Asymmetrical changes of gene expression, small RNAs and chromatin in two resynthesized wheat allotetraploids. Plant J. 2018;93:828-42.

43. Jiang J, Gill BS. Different species-specific chromosome translocations in Triticum timopheevii and T. turgidum support the diphyletic origin of polyploid wheats. Chromosom Res. 1994;2:59-64.

44. Patel RK, Jain M. NGS QC Toolkit: a toolkit for quality control of next generation sequencing data. PLoS One. 2012;7:e30619.

45. Li H, Handsaker B, Wysoker A, Fennell T, Ruan J, Homer N, Marth G, Abecasis $\mathrm{G}$, Durbin R, Proc GPD. The sequence alignment/map format and SAMtools. Bioinformatics. 2009;25:2078-9.

46. Rausch T, Zichner T, Schlattl A, Stutz AM, Benes V, Korbel JO. DELLY: structural variant discovery by integrated paired-end and split-read analysis. Bioinformatics. 2012;28:i333-9.

47. Layer RM, Chiang C, Quinlan AR, Hall IM. LUMPY: a probabilistic framework for structural variant discovery. Genome Biol. 2014;15:R84

48. Stuart T, Eichten SR, Cahn J, Karpievitch W, Borevitz JO, Lister R. Population scale mapping of transposable element diversity reveals links to gene regulation and epigenomic variation. Elife. 2016;5:e20777.

49. Trapnell C, Roberts A, Goff L, Pertea G, Kim D, Kelley DR, Pimentel H, Salzberg SL, Rinn JL, Pachter L. Differential gene and transcript expression analysis of RNA-seq experiments with TopHat and Cufflinks. Nat Protoc. 2012; 7:562-78

50. Conesa A, Gotz S, Garcia-Gomez JM, Terol J, Talon M, Robles M. Blast2GO: a universal tool for annotation, visualization and analysis in functional genomics research. Bioinformatics. 2005;21:3674-6.

51. Shiryev SA, Papadopoulos JS, Schaffer AA, Agarwala R. Improved BLAST searches using longer words for protein seeding. Bioinformatics. 2007;23:2949-51.

52. Krueger F, Andrews SR. Bismark: a flexible aligner and methylation caller for Bisulfite-Seq applications. Bioinformatics. 2011;27:1571-2.

53. Thorvaldsdottir H, Robinson JT, Mesirov JP. Integrative Genomics Viewer (IGV): high-performance genomics data visualization and exploration. Brief Bioinform. 2012;14:178-92.

54. Yuan J, Jiao W, Liu Y, Ye W, Wang X, Liu B, Song Q, Chen ZJ. Dynamic and reversible DNA methylation changes induced by genome separation and merger of polyploid wheat. Supplementary Datasets 2020. BIGD accession CRA002143 https://bigd.big.ac.cn/search/?dbld=\&q=CRA002143.

\section{Publisher's Note}

Springer Nature remains neutral with regard to jurisdictional claims in published maps and institutional affiliations.

Ready to submit your research? Choose BMC and benefit from:

- fast, convenient online submission

- thorough peer review by experienced researchers in your field

- rapid publication on acceptance

- support for research data, including large and complex data types

- gold Open Access which fosters wider collaboration and increased citations

- maximum visibility for your research: over $100 \mathrm{M}$ website views per year

At BMC, research is always in progress.

Learn more biomedcentral.com/submissions 